\title{
Representações sociais de profissionais de saúde da área hospitalar sobre o parto domiciliar planejado
}

Social representations of health professionals in the hospital area about planned home birth Representaciones sociales de los profesionales de la salud en el área hospitalaria acerca del parto planificado en el hogar

\section{Isabela Venturini Ayres Cunha', Júnia Aparecida Laia da Mata ${ }^{\mathrm{II}}$, Luciane Cristina Rodrigues Fernandes ${ }^{\mathrm{II}}$, Erika Zambrano Tanaka ${ }^{\mathrm{IV}}$, Clara Fróes de Oliveira Sanfelicev}

\begin{abstract}
Resumo: Objetivo: apreender as representações sociais de profissionais de saúde da área hospitalar sobre o parto domiciliar planejado. Método: estudo qualitativo, fundamentado na Teoria das Representações Sociais e realizado com 15 profissionais de saúde de um hospital de ensino. Os dados foram coletados no segundo semestre de 2019, por meio de entrevista semiestruturada. Utilizou-se a Análise Temática de Conteúdo. Resultados: os relatos originaram dois temas: 1) Parto domiciliar: uma possibilidade, com critérios de elegibilidade e 2) Parto domiciliar: uma miscelânea de conceitos, opiniões e interpretações. O grupo social investigado entende o parto domiciliar planejado como uma opção de assistência, com critérios de elegibilidade. No entanto, prevalece no senso coletivo uma associação desta modalidade de parto com situações de urgência/emergência, com potencial risco para mulheres e recém-nascidos. Conclusão: as representações compartilhadas revelam a generalização de informações sem respaldo científico sobre o parto domiciliar planejado.
\end{abstract}

Descritores: Saúde da mulher; Obstetrícia; Parto domiciliar; Enfermagem obstétrica; Pessoal de Saúde

Abstract: Objective: apprehending the social representations of health professionals in the hospital area about the planned home birth. Method: a qualitative study based on the Theory of Social Representations and conducted with 15 health professionals of a teaching hospital. Data were collected in the second half of 2019 through semi-structured interviews. Thematic Content Analysis was used. Results: the reports originated two themes: 1) Home birth: one possibility, with eligibility criteria and 2) Home birth: a miscellany of concepts, opinions and interpretations. The social

\footnotetext{
${ }^{\mathrm{I}}$ Enfermagem. Graduação. Faculdade de Enfermagem da Universidade Estadual de Campinas-FEnf/UNICAMP. Campinas, São Paulo, Brasil. Email: isaventurini15@gmail.com, Orcid: https://orcid.org/0000-0002-9711-0227

II Enfermagem Obstétrica. Doutorado. Faculdade de Enfermagem da Universidade Federal do Rio Grande do Sul - UFRGS. Porto Alegre, Rio Grande do Sul, Brasil. E-mail: junia.mata@ufrgs.br, Orcid: http://orcid.org/0000-0001-9062-8536

III Enfermagem Obstétrica. Mestrado. Faculdade de Enfermagem da Universidade Estadual de Campinas-FEnf/UNICAMP. Campinas, São Paulo, Brasil. E-mail: fernandeslcr@gmail.com, Orcid: https://orcid.org/0000-0002-5535-2383

IV Enfermagem Obstétrica. Doutorado. Faculdade de Enfermagem da Universidade Estadual de Campinas-FEnf/UNICAMP. Campinas, São Paulo, Brasil. E-mail: eztanaka@unicamp.br, Orcid: https://orcid.org/0000-0001-9913-2975

V Enfermagem Obstétrica. Doutorado. Faculdade de Enfermagem da Universidade Estadual de Campinas-FEnf/UNICAMP. Campinas, São Paulo, Brasil. E-mail: clarafos@unicamp.br, Orcid: http://orcid.org/0000-0003-1920-3193
} 
Representações sociais de profissionais de saúde da área hospitalar sobre o parto... | 2

group investigated understands planned home birth as an option of care, with eligibility criteria. However, a association of this mode of birth with urgent/emergency situations prevails in the collective sense, with potential risk for women and newborns. Conclusion: the shared representations reveal the generalization of information without scientific support about planned home birth.

Descriptors: Women's Health; Obstetrics; Home Childbirth; Obstetric Nursing; Health Personnel

Resumen: Objetivo: aprehender las representaciones sociales de profesionales de la salud en el área hospitalaria acerca del parto planificado en el hogar. Método: un estudio cualitativo basado en la Teoría de las Representaciones Sociales y realizado con 15 profesionales de la salud de un hospital escuela. Los datos fueron recolectados en el segundo semestre de 2019 a través de entrevistas semiestructuradas. Se utilizó el análisis de contenido temático. Resultados: los informes originaron dos temas: 1) Nacimiento en el hogar: una posibilidad, con criterios de elegibilidad y 2) Nacimiento en el hogar: una miscelánea de conceptos, opiniones e interpretaciones. El grupo social investigado entiende el parto planificado en el hogar como una opción de atención, con criterios de elegibilidad. Sin embargo, una asociación de este modo de parto con situaciones de urgencia/emergencia prevalece en el sentido colectivo, con riesgo potencial para las mujeres y los recién nacidos. Conclusión: las representaciones compartidas revelan la generalización de la información sin apoyo científico sobre el parto planificado en el hogar.

Descriptores: Salud de la Mujer; Obstetricia; Parto Domiciliario; Enfermería Obstétrica; Personal de Salud

\section{Introdução}

O parto domiciliar planejado é uma opção reconhecida pela Organização Mundial da Saúde (OMS), desde que a assistência seja realizada por um(a) profissional de saúde habilitado(a) (enfermeiras obstetras/obstetrizes) e qualificado(a). ${ }^{1}$ No Brasil, o Ministério da Saúde (MS) declara que, embora essa modalidade de assistência ao parto não faça parte das políticas atuais de saúde no país, o parto domiciliar planejado não deve ser desencorajado às multíparas de risco habitual, para as quais deve ser assegurado o acesso em tempo hábil e oportuno a uma maternidade, se houver necessidade de transferência. ${ }^{2}$ De acordo com a literatura atual, o parto domiciliar está relacionado a resultados maternos e fetais satisfatórios. Dentre eles, destaca-se a maior taxa de partos espontâneos e de satisfação materna, menores chances de intervenção e cirurgia cesariana, menor risco de distocia fetal e hemorragia pós-parto, além de percentuais semelhantes para morbimortalidade neonatal, quando comparados os ambientes hospitalar e domiciliar. ${ }^{3-6}$

$\mathrm{Na}$ ótica das mulheres, a insatisfação com o modelo obstétrico hospitalar vigente é um dos principais motivos que justifica a escolha pelo parto domiciliar planejado. No modelo hospitalar, as usuárias dos serviços percebem que o parto é visto como um processo patológico, sendo atendido de 
3 | Cunha IVA, Mata JAL, Fernandes LCR, Tanaka EZ, Sanfelice CFO.

forma despersonalizada e impositiva, no que se refere às rotinas institucionais. Logo, neste cenário de grande vulnerabilidade materna, as condutas, por vezes, podem ser tomadas de forma arbitrária, sem considerar o diálogo e as decisões maternas. ${ }^{4-7}$

Diante desse contexto, as estatísticas mostram uma tendência de crescimento na busca pela opção do parto domiciliar planejado, o que pode ser observado ao serem analisadas as taxas nos âmbitos nacional e internacional. Nos Estados Unidos, por exemplo, a taxa de parto domiciliar planejado aumentou 77\% de 2004 a $2017^{8}$ e, no Brasil, observa-se o mesmo fenômeno nas regiões mais urbanizadas do país (Sul, Sudeste e Centro-Oeste) no período de 2010 a 2017.9

É importante ressaltar que, no Brasil, a categoria da enfermagem obstétrica é a profissão que adere de forma majoritária a este modelo de atendimento. ${ }^{10}$ Dessa maneira, as informações relacionadas ao parto domiciliar planejado repercutem diretamente no trabalho das(os) enfermeiras(os) obstetras, o que justifica o aprofundamento na investigação sobre o tema e suas interfaces. Embora exista uma vasta produção internacional no assunto, uma recente revisão da literatura sobre o parto domiciliar planejado mostrou que a produção nacional nesta temática ainda apresenta lacunas, pois retrata, principalmente, os desfechos maternos e neonatais deste tipo de assistência/serviço. ${ }^{4}$

Nesse sentido, considera-se que há necessidade de aprofundar o entendimento sobre o parto domiciliar planejado para além das questões técnicas, permitindo a compreensão do fenômeno na ótica de outros atores sociais, por exemplo, os profissionais de saúde que não atuam na assistência ao parto em domicílio. Para isso, este estudo foi fundamento na Teoria das Representações Sociais (TRS), proposta pelo psicólogo social francês Serge Moscovici, nos anos 1960. ${ }^{11}$

A TRS pode ser compreendida como uma forma de conhecimento socialmente elaborado e compartilhado, com um objetivo prático, e que contribui para a construção de uma realidade comum ao conjunto social. ${ }^{11}$ Esse movimento permite a formação de grupos que partilham das mesmas concepções sobre o assunto e, portanto, adotam condutas e práticas semelhantes. ${ }^{12}$

A articulação dos conceitos da TRS com o campo da obstetrícia mostra que a ideia de parto e 
Representações sociais de profissionais de saúde da área hospitalar sobre o parto... $\mid 4$

nascimento predominante, que corresponde ao universo consensual, é a do parto institucionalizado, atendido pelo médico e com utilização maciça de intervenção e tecnologia em todo o processo. Ou seja, o pensamento compartilhado no senso comum é de que o parto e o nascimento devem ocorrer no hospital, de preferência com o mínimo de “dor e sofrimento” para a mulher. Já a opção pelo parto domiciliar planejado pode ser compreendida como fato não familiar do universo consensual (senso comum), gerador de tensão e desconforto. ${ }^{7}$

Diante do exposto, o estudo visa responder à seguinte questão de pesquisa: Quais são as representações sociais de profissionais de saúde da área hospitalar sobre o parto domiciliar planejado? Acredita-se que esta investigação tenha potencial para ampliar conhecimentos sobre o objeto de estudo e promover a capilarização das discussões sobre o parto domiciliar planejado entre diferentes profissionais de saúde, bem como agregar ao saber da enfermagem obstétrica. Neste sentido, o objetivo deste estudo foi apreender as representações sociais de profissionais de saúde da área hospitalar sobre o parto domiciliar planejado.

\section{Método}

Utilizou-se a TRS para o embasamento teórico deste estudo. Ela tem sido empregada na área de enfermagem, devido à possibilidade de o pesquisador(a) captar a interpretação dos próprios participantes da realidade que se almeja pesquisar, permitindo a compreensão das atitudes e comportamentos que um determinado grupo social tem frente a um objeto psicossocial. ${ }^{12}$

Quando as representações sociais são focadas como produto, como no caso deste estudo, a pesquisa visa depreender os elementos constitutivos das representações (informações, imagens, opiniões e crenças), tendo como referência as condições sociais de sua produção. Quando se concentra como processo, a pesquisa volta-se à compreensão da elaboração e transformação das representações sob a força das determinações sociais. ${ }^{12}$

Trata-se de uma pesquisa qualitativa, exploratória e descritiva. O público-alvo incluiu profissionais de saúde que trabalhavam em um hospital universitário público, localizado no interior 
5 | Cunha IVA, Mata JAL, Fernandes LCR, Tanaka EZ, Sanfelice CFO.

do Estado de São Paulo. Este hospital atende em média 230 partos/mês, e é referência para gestantes de risco habitual e alto risco de seis municípios da região. Foram definidos os seguintes critérios de seleção: profissionais graduados(as) em medicina ou enfermagem, com especialização em obstetrícia ou neonatologia; alocados em uma das três unidades (Centro Obstétrico, Alojamento Conjunto e/ou Unidade de Terapia Intensiva Neonatal) da instituição cenário do estudo.

O número de participantes foi definido utilizando-se o conceito de "ponto de saturação”, empregado em investigações qualitativas. Este ponto é alcançado quando os novos participantes passam a repetir informações mencionadas em entrevistas anteriores e o acréscimo de novos conteúdos não altera a compreensão do fenômeno estudado. ${ }^{13}$

O grupo a ser estudado foi definido por conveniência, sendo o primeiro entrevistado indicado pela coordenação das unidades, e os(as) demais participantes foram recrutados a partir da técnica de amostragem por bola de neve (Snowball Sampling). Nela, os primeiros entrevistados indicam os próximos, que, por sua vez, indicarão outros e assim por diante, ${ }^{14}$ até o momento em que as novas informações passam a ser repetidas (ponto de saturação). ${ }^{13}$

Durante a coleta, não houve recusa à participação. Os dados foram levantados no segundo semestre de 2019, por meio de um roteiro semiestruturado, composto por duas partes: 1- Perfil sociodemográfico; e 2- Entrevista semiestruturada, contendo três perguntas abertas disparadoras: a) Qual a sua opinião sobre o parto domiciliar planejado?; b) Na sua opinião, toda mulher pode parir em casa?; c) Você acha que esse tipo de parto pode trazer benefícios e/ou malefícios para a mãe e o bebê? Para validar o instrumento utilizado, foi realizado um teste piloto com quatro profissionais de saúde de outra instituição, sendo necessário o ajuste semântico de apenas uma pergunta.

Os participantes foram convidados a participar da pesquisa nas instalações do hospital, nos dias em que estavam de plantão. Inicialmente, foram apresentados os objetivos do estudo e feito o convite. As entrevistas foram realizadas em um ambiente reservado da instituição, no momento em que o profissional julgou pertinente, sem comprometimento da rotina de trabalho.

As entrevistas duraram, em média, 10 minutos, foram audiogravadas com gravador digital e, 
Representações sociais de profissionais de saúde da área hospitalar sobre o parto... |6

imediatamente após a coleta, transcritas pela pesquisadora principal. As transcrições foram apresentadas aos participantes, na semana seguinte ao levantamento, para validação e/ou correções.

Os dados foram analisados segundo a Análise Temática de Conteúdo ${ }^{15}$, a qual compreende uma técnica de tratamento dos dados que visa obter, por meio de procedimentos sistemáticos, indicadores que permitam a inferência de conhecimentos relativos às mensagens e o desvelamento das relações que se estabelecem além das falas propriamente ditas. Esse referencial metodológico prevê três fases fundamentais, as quais foram criteriosamente seguidas: 1) pré-análise; 2) exploração do material; e 3) tratamento dos resultados, inferência e interpretação. Na etapa de pré-análise, o material foi organizado para que se tornasse operacional. Esse processo foi realizado quatro fases: leitura flutuante; escolha dos documentos; elaboração de hipóteses/objetivos; e, por último, referenciação dos índices e elaboração dos indicadores. Em seguida, procedeu-se à exploração do material, realizando-se a codificação, classificação e categorização por temas. Por fim, a condensação e o destaque das informações para análise, culminando nas interpretações inferenciais, inerentes à fase de tratamento dos resultados, inferência e interpretação. ${ }^{15}$

Visando a qualidade e transparência da redação do relatório, referente ao rigor metodológico da investigação e à credibilidade da pesquisa, utilizou-se o checklist Consolidated Criteria for Reporting Qualitative Studies (COREQ). Para garantir o anonimato, os entrevistados foram identificados pela letra "E” seguida de um número aleatório. O estudo seguiu as diretrizes e foi conduzido de acordo com os padrões éticos exigidos para pesquisas que envolvem seres humanos dispostos nas Resoluções 466/2012 e 510/2016 do Conselho Nacional de Saúde, obtendo aprovação, em 30 de abril de 2019, pelo Comitê de Ética em Pesquisa, sob o protocolo no 3.294 .813 . Todos os participantes assinaram o Termo de Consentimento Livre e Esclarecido (TCLE).

\section{Resultados}

Participaram da pesquisa 15 profissionais de saúde, incluindo médicos(as) e enfermeiros(as) com diferentes especializações, conforme mostra a tabela abaixo: 
7 | Cunha IVA, Mata JAL, Fernandes LCR, Tanaka EZ, Sanfelice CFO.

Tabela 1- Profissão e especialização dos participantes da pesquisa. Campinas/SP, 2019

\begin{tabular}{llcc}
\hline Profissão & Especialidade & N & \% \\
\hline Médico(a) & Obstetrícia & 5 & 33,4 \\
& Neonatologia & 2 & 13,3 \\
Enfermeiro(a) & Obstetrícia & 6 & 40,0 \\
& Neonatologia & 2 & 13,3 \\
Total & & 15 & 100
\end{tabular}

Dentre os participantes, $13(86,6 \%)$ eram do sexo feminino e dois $(13,4 \%)$, do sexo masculino, a idade média foi de 35 anos, variando no intervalo de 25 a 58 anos; o tempo médio de trabalho na instituição foi de nove anos e todos possuíam ensino superior completo. Com relação à cor, 13 $(86,6 \%)$ se autodeclaram brancos e dois $(13,4 \%)$, pardos.

Nenhum entrevistado possuía experiência/vivência com o atendimento ao parto domiciliar planejado, mas alguns participantes citaram situações em que mulheres chegaram transferidas de casa para o hospital, incluindo situações planejadas e aquelas não planejadas, experiências que, provavelmente, interferiram na construção das opiniões expressadas.

A análise dos dados permitiu a construção de dois temas que foram nomeados como: a) Parto domiciliar: uma possibilidade, com critérios de elegibilidade; e b) Parto domiciliar: uma miscelânea de conceitos, opiniões e interpretações. A seguir, serão apresentados os depoimentos que constituem os temas supracitados.

\section{Parto domiciliar: uma possibilidade, com critérios de elegibilidade}

Uma parcela dos participantes demonstrou entender que o parto domiciliar é uma opção viável às mulheres, desde que sejam adotados alguns critérios de elegibilidade. Dentre os mencionados, a estratificação do risco gestacional apareceu como o mais relevante.

Acho que existe critério! [...] depende da gestação, do pré-natal, da condição materna, é multifatorial! Acho que não são todas [as mulheres] que podem não!(E4)

Desde que ela tenha todas as condições favoráveis, que ela não tenha nenhuma comorbidade e que esteja tudo bem com o pré-natal dela. (E12)

Se for um pré-natal de baixo risco, sem nenhuma alteração durante a gravidez. Alto risco não tem a possibilidade de se arriscar assim. (E2)

Não dá para ser um pré-natal mal feito ou que não teve nenhuma avaliação da mãe e do bebê, e só achar que vai nascer em casa que vai dar tudo certo. Tem risco de dar errado. (E14) 
Representações sociais de profissionais de saúde da área hospitalar sobre o parto... $\mid 8$

Não acho que toda mulher poderia parir em casa. Acho que tem vieses, risco no pré-natal, risco de ser um bebê de risco. Não, toda [mulher] não. Mães de baixo risco. As de alto risco precisam ser adequadamente acompanhadas em um serviço adequado. (E3)

Os depoimentos demonstram que os profissionais reconheciam a existência de critérios préestabelecidos para as mulheres que tenham intenção de vivenciar o parto domiciliar planejado. Outras variáveis que se relacionam aos critérios de elegibilidade foram desveladas.

[...] se for para ter um parto domiciliar, desde que tenha uma equipe de apoio, desde que tenha uma retaguarda. (E11)

Esse tipo de parto precisa de uma preparação [...]. Uma estrutura, uma equipe preparada, que saiba conduzir esse parto, que saiba agir caso alguma coisa não saia como o planejado. Uma orientação para essa gestante, para que ela saiba também tudo o que pode acontecer, estar preparada para essas situações. E suporte! Da familia, dos profissionais que estão atendendo esta mulher, do parceiro. Acho que o mais importante é esta estrutura. (E8)

Acho que são necessários critérios. Explicar para essa mulher certinho o porquê ela não pode parir em casa. E, se sim, há a necessidade de um preparo profissional, de entender que pode acontecer alguma coisa errada e estar preparada para tomar atitude. Avaliar o percurso até o hospital, se é uma coisa viável. Tudo tem que ser bem estruturado e a equipe preparada. (E8)

Tudo precisa ser conversado e é necessário que haja uma decisão conjunta entre o casal e a equipe, que deve ser preparada e estruturada para atender essa gestante. (E3)

Por meio das entrevistas, identifica-se que os participantes elencaram critérios relacionados às questões de logística, como estrutura para o atendimento e treinamento dos profissionais envolvidos, e emocionais, como preparação da mulher e apoio familiar. Esses foram entendidos como importantes fatores a serem considerados na escolha pelo parto domiciliar planejado.

\section{Parto domiciliar: uma miscelânea de conceitos, opiniões e interpretações}

Com exceção da temática sobre os critérios de elegibilidade, a qual foi praticamente unânime entre os participantes, o restante dos depoimentos demonstrou uma miscelânea entre conceitos, opiniões pessoais e interpretações questionáveis sobre o parto domiciliar planejado. No que se refere aos riscos e à atuação da equipe no domicílio, foram citadas situações como:

Não é que eu acho, eu tenho certeza que [o parto domiciliar] implica riscos para a gestante e para o bebê. Risco de hemorragia, risco de uma assistência mais demorada ao recém-nascido, no caso de necessidade de reanimação. (E6)

Eu acho que no domicilio, se tiver alguma intercorrência tanto com o bebê como com a mãe, 
9 | Cunha IVA, Mata JAL, Fernandes LCR, Tanaka EZ, Sanfelice CFO.

você não tem tempo hábil de tomar uma providência. Então, você acaba colocando tanto a mãe quanto o bebê em risco. (E8)

Então tem alguns casos em particular que eu acho que, se a mulher parir em casa, esse tempo de deslocamento para o hospital pode comprometer o futuro da criança. (E3)

Acho que um risco de anóxia, se não tiver um pediatra para atender o bebê. Uma mãe que possa ter uma convulsão, um choque hipovolêmico, demorar para ser atendida e eventualmente ir a óbito. (E13)

A gestante tem um risco de evoluir para uma hipotonia ou atonia uterina, vai aumentar muito o sangramento dela, e se não conseguisse ser revertida com massagem? Ela precisaria rapidamente ter acesso venoso pego, transfusão de sangue e talvez até uma histerectomia. (E2) E se o bebê precisar de VPP [ventilação por pressão positiva], precisar de qualquer cuidado, [...] de um oxigênio. Você não vai colher a gaso [gasometria] rapidamente para ver se ele anoxiou ou não. Perde tanto parâmetro para saber se ele ficou bem, se vai ficar bem ou não. Então, meio que você perde a chance de reverter aquilo. (E2)

Eu sou contrário ao parto domiciliar planejado porque eu acredito que, em um ambiente hospitalar, a paciente tem uma maior segurança. Tanto a mãe como o bebê. Porque, por mais que o parto seja uma situação fisiológica que numa situação de baixo risco a tendência é ocorrer tudo bem, mas sempre tem riscos envolvidos, então, na minha opinião o parto tem que ser sempre hospitalar. (E6)

Uma distocia, o bebê desce e você auscultar uma bradicardia e a gente sabe que esse tempo de bradicardia já mostra que é uma acidose para criança. Essa bradicardia gera uma acidose e, se você não está no ambiente hospitalar, essa acidose compromete o sistema nervoso dessa criança. Se você não fizer uma intervenção rápida. Seja um fórceps, seja uma cesárea. (E3)

O que a gente fica preocupada no parto domiciliar é se vai ter uma assistência adequada, assim como é no hospital. (E5)

Se for planejado, com um médico, com recursos para o bebê e para a mãe e se tiver uma ambulância de UTI[Unidade de Terapia Intensiva] na porta. (E13)

Não. Na minha opinião, toda mulher deveria parir no hospital.(E6)

Estes depoimentos desvelam que para os profissionais de saúde o parto domiciliar planejado é como um evento de eminente risco para a mãe e o feto, com predominância de situações de urgência/emergência, as quais acabam fundamentando os argumentos contrários a essa modalidade de assistência ao parto. Também aparecem os questionamentos sobre a conduta e atuação da equipe durante a assistência desempenhada no parto domiciliar planejado.

\section{Discussão}

Os depoimentos demonstraram que alguns profissionais de saúde entendiam o parto domiciliar planejado como uma opção viável, desde que sejam considerados critérios de elegibilidade para a sua ocorrência. Isso demonstra que os participantes reconheceram um dos 
Representações sociais de profissionais de saúde da área hospitalar sobre o parto... $\mid 10$ principais pilares que sustentam esta modalidade de assistência ao parto, pois, segundo a literatura, a gestação de risco habitual é descrita como pré-requisito essencial para a segurança do parto domiciliar planejado., ${ }^{210,16-18}$

Os critérios de elegibilidade são importantes e devem ser seguidos rigorosamente, já que a existência de qualquer condição de saúde que aumente o risco materno ou fetal torna a opção de parto domiciliar perigosa para ambos. ${ }^{18}$ Outro aspecto revelado nas entrevistas se relaciona à preocupação do grupo estudado com a competência e o preparo técnico dos profissionais que atendem o parto domiciliar planejado, para que seja garantida a adequada atuação destes frente a possíveis intercorrências maternas e neonatais.

Considerando esse aspecto, a literatura mostra que, para o atendimento de um parto em casa, é necessário que o profissional seja capacitado para atuar nas urgências e emergências obstétricas e neonatais e saiba identificar possíveis complicações obstétricas que necessitem de encaminhamento para um serviço de referência. ${ }^{18-19}$ São fundamentais a experiência técnica prévia dos profissionais envolvidos nessa modalidade de atendimento ao parto e nascimento e o treinamento para atuação em situações de urgência/emergência, bem como a constante atualização teórico-prática. Entende-se que estes requisitos são promotores de um cuidado seguro e qualificado.

Em relação aos profissionais habilitados no atendimento ao parto domiciliar planejado, destaca-se a enfermeira obstetra. Uma vez que esta modalidade de atendimento vem crescendo no Brasil e que o número enfermeiras obstetras que se dedicam a esta assistência aumentou significativamente, ${ }^{10}$ é importante salientar que essas profissionais possuem respaldo legal e são habilitadas para exercer o atendimento pré-natal e a assistência ao parto de risco habitual em ambiente domiciliar. ${ }^{10,20-21}$

No domicílio, essas especialistas devem ter habilidades específicas para realizar uma avaliação contínua do processo parturitivo, tais como saber identificar precocemente os sinais de risco, oferecer suporte básico de vida e estabilizar as mulheres e os neonatos, até a transferência hospitalar, nos casos de intercorrências. ${ }^{2,5}$ Tais habilidades são cruciais na garantia de segurança do 
11 | Cunha IVA, Mata JAL, Fernandes LCR, Tanaka EZ, Sanfelice CFO.

cuidado à saúde materno-fetal e, por isso, podem ser questionadas pelas mulheres/famílias que planejam o parto domiciliar.

Apesar de os depoimentos demonstrarem preocupação com a qualificação profissional para o atendimento, não houve menção sobre qual profissional de saúde pode atuar neste cenário. Sobre este aspecto, é importante destacar que o Conselho Federal de Medicina, desde 2012, recomenda que a realização do parto ocorra, preferencialmente, em ambiente hospitalar. ${ }^{22}$

Esse é um aspecto que deveria ser problematizado e discutido entre os conselhos profissionais, uma vez que a prática assistencial deve ser sempre guiada pelas recomendações científicas mais recentes. Dessa maneira, poderiam ser evitadas a construção e a difusão de mitos e posicionamentos desalinhados à ciência e que afetam a liberdade de escolha das mulheres/famílias sobre o local de nascimento de seus filhos.

Dentre as representações desveladas, identificou-se preocupação por parte dos profissionais de saúde com a decisão compartilhada entre a equipe de atendimento e a mulher/família ou o casal. Esse é um importante aspecto na compreensão sobre o parto domiciliar planejado, já que a escolha pelo local de parto deve ser entendida como um direito reprodutivo básico, que respeita a autonomia e o protagonismo materno..$^{10,17-18,23}$

Por isso, é necessário que as mulheres recebam informações precisas e livres de preconceitos, que contemplem os riscos e os benefícios de cada tipo de parto, a probabilidade de serem transferidas para uma maternidade, as razões que justificariam esta medida e o tempo necessário para tal, para que possam fazer suas escolhas de forma consciente e informada., ${ }^{2,17-21}$ Também é importante que as mulheres compreendam e reconheçam os limites da assistência ao parto em domicílio, o que se caracteriza como uma estratégia para aumentar a segurança neste cenário. ${ }^{18}$

A decisão pelo parto domiciliar planejado deve ser formalizada por meio de um documento, que pode ser um contrato elaborado em comum acordo entre as partes, constando a ciência e a assinatura dos interessados. Esse contrato, denominado TCLE, busca garantir o registro das informações discutidas entre a equipe e a mulher/família ou o casal, quais as condutas a serem tomadas no caso de 
intercorrências/complicações obstétricas e/ou neonatais, incluindo ações que serão realizadas em situação de transferência eletiva ou de emergência para um serviço de saúde de referência. ${ }^{20-21}$

Os depoimentos revelaram que o parto domiciliar planejado é representado como um evento potencialmente arriscado para a mulher e o seu bebê, com eminência de situações de urgência ou emergência. Desse modo, os participantes demonstraram preocupação com o percurso até o hospital e com a necessidade de retaguarda durante o atendimento no domicílio. Sobre esse quesito, tanto o MS, como as diretrizes internacionais de países que mantêm o parto domiciliar integrado ao sistema de saúde endossam que as mulheres que optarem por parir no domicílio devem ter assegurado o acesso em tempo hábil e oportuno a uma maternidade.,17-19 Essa é uma importante medida a ser considerada no momento da escolha pelo parto domiciliar planejado, uma vez que, diante de situações de urgência e/ou emergência, o tempo gasto no trajeto entre a casa e o hospital pode ser um fator de agravamento e desfecho negativo.

De acordo com a literatura científica internacional, alguns componentes garantem melhores resultados nos partos domiciliares planejados, a saber: a) ótima integração dos profissionais de atendimento ao sistema de saúde; b) comunicação efetiva entre o hospital e as equipes de atendimento; c) sinalizações específicas para o transporte de emergência; e d) um plano préestabelecido para o encaminhamento a um hospital, em caso de complicações. ${ }^{17-18}$ A comunicação efetiva e antecipada entre equipes de atendimento e serviços de saúde se justifica por diversos motivos. Inicialmente, porque o gerenciamento de transferências pode ser muito favorecido se o hospital já possui informações prévias sobre a mulher a ser atendida (ou o bebê, em caso de transferência neonatal).

Informar ao hospital sobre uma emergência clínica permite que o serviço antecipe a sua chegada e organize o atendimento de modo que o cuidado às parturientes internadas não seja negativamente impactado ${ }^{18}$ e a assistência à mulher e/ou ao bebê transferidos do parto domiciliar seja efetiva. Tendo em vista que o parto domiciliar planejado não faz parte das políticas de saúde do Brasil, diferentemente de países como Reino Unido, Estados Unidos, Canadá e Dinamarca, ${ }^{24}$ por 
13 | Cunha IVA, Mata JAL, Fernandes LCR, Tanaka EZ, Sanfelice CFO.

exemplo, pressupõe-se que a comunicação necessária entre equipe de atendimento no domicílio e instituição de saúde integrada à rede de atenção à saúde apresente fragilidades no contexto do país.

É importante ressaltar que algumas enfermeiras obstetras, geralmente, trabalham em parceria com um profissional médico obstetra na retaguarda, que é chamado de backup. Esse trabalho em equipe multiprofissional não tem o intuito de "supervisionar" as atividades das enfermeiras obstetras, mas, sim, garantir a segurança, caso o binômio necessite de uma intervenção, assistência medicamentosa e/ou invasiva no nível hospitalar, podendo este receber a mulher e/ou o bebê e dar continuidade ao atendimento. ${ }^{10}$

Além da desarticulação entre equipes e instituições de saúde, as profissionais que atendem o parto domiciliar planejado no país enfrentam outros desafios, tais como preconceitos relacionados à falta de informações, retaliações em situações de transferências hospitalares e dificuldades na aquisição de documentos (Declaração de Nascidos Vivos) e insumos de trabalho. ${ }^{23}$ Tais desafios podem ocorrer pelo fato de esta modalidade de assistência ao parto permanecer à margem do sistema de saúde do Brasil, sem uma regulamentação específica. ${ }^{10,20}$ O grupo social investigado acredita que existem critérios de elegibilidade para o parto domiciliar planejado, bem como fatores que precisam ser levados em consideração. Essa visão sobre o parto domiciliar planejado é satisfatória, tendo em vista que estes profissionais estavam inseridos em um serviço de saúde que pode receber mulheres provenientes desta modalidade de nascimento e, portanto, poderiam prestar um atendimento livre de preconceitos diante da escolha da mulher/família ou do casal.

Por outro lado, o parto domiciliar planejado permanece representado pelos participantes como um evento que envolve mais riscos do que o parto hospitalar. Sobre este aspecto, pesquisas apontam que, no cenário domiciliar, as mulheres estão expostas a menos riscos, pois estão menos propensas a receberem intervenções medicamentosas, como indução do parto e analgesia farmacológica, ${ }^{3,6,25}$ a sofrerem episiotomia ${ }^{25}$ e lacerações perineais de terceiro ou quarto grau. ${ }^{25}$ Além disso, no parto domiciliar planejado é menos provável a ocorrência de complicações do trabalho de parto, ${ }^{6}$ hemorragia, ${ }^{3,25}$ infecção pós-parto, ${ }^{25}$ complicação fetal ${ }^{3}$ e reanimação neonatal. ${ }^{6}$ 
Representações sociais de profissionais de saúde da área hospitalar sobre o parto... | 14

A prática de um modelo de atenção intraparto minimamente intervencionista é um dos aspectos responsáveis pelos bons resultados na assistência ao parto domiciliar planejado, especialmente quando associada à cuidadosa triagem das mulheres e à adequada integração deste modelo com o sistema de saúde local. ${ }^{26}$ Em virtude desses achados, infere-se que as representações sociais sobre o parto domiciliar planejado dos profissionais estudados encontram-se em desacordo com o que demonstram as atuais produções científicas sobre o tema.

Nesse sentido, entende-se que existe uma fragilidade na compreensão da causalidade da maioria das complicações do processo parturitivo. Mesmo que possam ocorrer fatalidades durante um atendimento, sugere-se que o modelo assistencial de caráter intervencionista, muito praticado no ambiente hospitalar, é um fator crucial na gênese de complicações e na consequente necessidade de cuidados avançados, o que não se aplica para o ambiente doméstico. $\mathrm{Na}$ contramão do modelo assistencial praticado no parto domiciliar planejado, o parto hospitalar faz pouco uso das tecnologias que favorecem a progressão fisiológica do trabalho de parto e utiliza, de forma excessiva e muitas vezes desnecessária, intervenções que geram dor e sofrimento. ${ }^{25}$ Essas intervenções, quando aplicadas rotineiramente ou sem respaldo científico, estão associadas a resultados maternos e perinatais desfavoráveis. ${ }^{27}$

A literatura científica reforça que o cuidado intraparto com o mínimo de intervenção representa um dos pilares para uma experiência de parto positiva, ${ }^{26}$ o que pode contribuir com o alto índice de satisfação materna com a experiência do parto domiciliar planejado. Dessa maneira, é emergente a necessidade de compreensão do possível fenômeno de causa-efeito que envolve o uso abusivo de intervenções e as consequentes intercorrências na assistência ao parto.

O entendimento dessa problemática pode contribuir para desconstruir a concepção, predominante no senso comum, de que as mulheres que optam por parir em casa estão se colocando em eminente situação de risco. Isto porque o parto domiciliar planejado é um modelo de assistência menos intervencionista que, consequentemente, torna as parturientes e seus fetos menos sujeitos às complicações. Foi possível verificar que existe uma crença comum entre os profissionais de saúde 
15 | Cunha IVA, Mata JAL, Fernandes LCR, Tanaka EZ, Sanfelice CFO.

de que as situações de urgência e emergência não conseguem ser bem manejadas no ambiente domiciliar (falta de tempo hábil para tomar uma providência, assistência mais demorada, ausência de insumos básicos, perda de tempo durante as transferências, etc.). É válido salientar que não foram encontrados estudos científicos que investiguem o manejo das situações de urgência e emergência durante o atendimento a um parto domiciliar planejado, que poderiam ser utilizados para afirmar ou refutar tais dados levantados nas entrevistas, o que torna este assunto nebuloso e favorece a construção de diversos cenários no imaginário comum.

Até o presente momento, foi identificada somente uma publicação nacional que apresenta e descreve a organização do serviço do parto domiciliar planejado. ${ }^{20}$ Assim, é emergente a necessidade de delineamento e divulgação de diretrizes e/ou protocolos específicos sobre este tipo de assistência, com intuito de clarear de forma minuciosa o processo de trabalho desenvolvido. Nessas diretrizes (ou em protocolos) poderiam ser incluídos aspectos como: instrumentos e insumos de trabalho utilizados; fluxo de atendimento; medidas a serem tomadas nas situações de urgência/emergência; perfil e pré-requisitos dos profissionais que prestam esse atendimento, entre outros detalhes que possam favorecer a compreensão e operacionalização do parto em casa.

Sobre a questão do tempo gasto nas situações de transferência hospitalar, um único estudo realizado na Inglaterra se propôs a estimar a duração das transferências em partos domiciliares planejados e seus efeitos nos resultados neonatais. ${ }^{28}$ Os pesquisadores encontraram que o tempo médio de transferência, o que inclui o período entre a tomada de decisão e o primeiro atendimento no serviço de saúde, foi de 49 minutos. Nas situações prévias ao nascimento, consideradas como potencialmente urgentes, esse tempo diminuiu cerca de 8-10 minutos, com mediana de 42 minutos. A maioria das transferências não era de caráter urgente e as emergências e os resultados adversos foram incomuns. Com relação ao desfecho neonatal, foram encontrados resultados adversos em 1$2 \%$ das mulheres que deram à luz em até 60 minutos de transferência. ${ }^{28}$

Os achados da literatura mostram que a transferência para o hospital não é uma condição frequente nos partos domiciliares planejados, o que é o oposto do representado pelos profissionais 
Representações sociais de profissionais de saúde da área hospitalar sobre o parto... | 16 de saúde participantes deste estudo.

No Brasil, uma revisão sistemática nacional sobre o parto domiciliar apontou uma taxa de transferência materna que varia em torno de 7,4 a 20\%, ${ }^{4}$ abaixo ou em torno do que é apresentado em estudo internacional (54\% para primíparas e $14 \%$ para multíparas). ${ }^{29}$ Com relação à transferência de recém-nascidos, esta revisão detectou taxas entre 0,7 e $1 \%,{ }^{4}$ e nenhuma transferência aconteceu em decorrência dos cuidados prestados ao parto ou do processo parturitivo em si, e nenhum RN foi internado em Unidade de Terapia Intensiva (UTI) neonatal. Os motivos das transferências foram arritmia cardíaca e epidermólise bolhosa não diagnosticada anteriormente ao parto. ${ }^{4}$

Outro estudo nacional, realizado no Distrito Federal, apresentou taxas semelhantes, sendo $21,1 \%$ a de transferência materna e 1,2\% a de recém-nascidos, e nenhum dos casos apresentou desfecho negativo após a transferência. ${ }^{5}$ Essas informações desconstroem a crença de que o parto domiciliar planejado possui frequentes intercorrências neonatais. Ela é, de fato, sem fundamentação científica e precisa ser problematizada nas discussões sobre a temática.

Entende-se que os resultados desta pesquisa possuem limitações relacionadas à regionalização da amostra. Por isso, defende-se a necessidade de realização de investigações futuras que incluam hospitais de ensino (ou não) localizados em diferentes regiões do Brasil.

Além disso, a abordagem de apenas duas categorias de profissionais da saúde também se configurou como uma restrição deste trabalho. Entretanto, foi dado o passo inicial por um caminho que pode ser percorrido com novas investigações que ampliem amostras e cenários, com foco no objeto de estudo tratado.

\section{Conclusão}

Para o grupo social investigado nesta pesquisa, profissionais de saúde de um hospital de ensino, o parto domiciliar planejado é uma possibilidade de assistência à parturiente, desde que sejam atendidos alguns critérios de elegibilidade. No entanto, prevalece uma forte crença de que 
17 | Cunha IVA, Mata JAL, Fernandes LCR, Tanaka EZ, Sanfelice CFO.

este evento é potencialmente mais perigoso para as mulheres e seus recém-nascidos em comparação com a assistência hospitalar, estando o binômio sujeito a situações de urgência e emergência eminentes.

As opiniões compartilhadas no grupo demonstram ausência de clareza sobre aspectos do processo de trabalho na assistência ao parto no domicílio, tais como treinamento e habilitação dos profissionais envolvidos, riscos maternos e fetais na assistência, logística de trabalho e manejo clínico nas situações de urgência e emergência. Percebeu-se que opiniões reveladas não encontram sustentação na literatura científica disponível na atualidade, o que impacta de forma negativa no trabalho realizado pelas enfermeiras obstetras e suas equipes de atendimento.

A limitada produção nacional e divulgação de evidências científicas sobre o parto domiciliar planejado pode justificar a ausência de clareza sobre o tema para os participantes do estudo. Isto reforça a necessidade de mais pesquisas sobre essa temática, com objetivo de romper com determinadas crenças e mitos e aumentar a visibilidade dessa modalidade de assistência à saúde.

Por fim, considera-se que este estudo agrega conhecimento ao campo da enfermagem por elucidar representações sociais de duas categorias profissionais envolvidas na atenção obstétrica e que podem influenciar no contexto da assistência ao parto domiciliar planejado, já que transferências são previstas e podem ocorrer no atendimento a esse tipo de parto. As representações desveladas podem ampliar reflexões e discussões no campo do ensino, no meio científico e na prática profissional, abrindo caminho para a compreensão dos desafios enfrentados pelas enfermeiras que prestam este serviço, bem como para a construção de estratégias para manejá-los.

\section{Referências}

1. World Health Organization (WHO). Strengthening quality midwifery education for Universal Health Coverage 2030: working together to ensure quality care for all mothers and newborns [Internet]. Geneva: Word Health Organization; 2019 [cited 2019 Oct 29]. Available from: https://www.who.int/maternal_child_adolescent/topics/quality-of-care/midwifery/strengthening-midwiferyeducation/en/ 
2. Ministério da Saúde (BR), Secretaria de Ciência, Tecnologia e Insumos Estratégicos, Departamento de Gestão e Incorporação de Tecnologias em Saúde. Diretrizes nacionais de assistência ao parto normal: versão resumida [Internet]. Brasília (DF): Ministério da Saúde; 2017 [acesso em 2019 out 29]. Disponível em: https://bvsms.saude.gov.br/bvs/publicacoes/diretrizes_nacionais_assistencia_parto_normal.pdf

3. Rossi AC, Prefumo F. Planned home versus planned hospital births in women at low-risk pregnancy: a systematic review with meta-analysis. Eur J Obstet Gynecol Reprod Biol. 2018; 222:102-8. doi: 10.1016/j.ejogrb.2018.01.016

4. Cursino TP, Benincasa M. Parto domiciliar planejado no Brasil: uma revisão sistemática nacional. Ciênc Saúde Colet. 2020;25(4):1433-44. doi: 10.1590/1413-81232020254.13582018

5. Santos SS, Boeckmann LMM, Baraldi ACP, Melo MC. Resultados de partos domiciliares planejados assistidos por enfermeiras obstétricas. Rev Enferm UFSM. 2018;8(1):129-43. doi:10.5902/2179769228345

6. Doherty D, Nathan L, Hutchinson M, Somerville S, Hauck Y, Hornbuckle J. Planned home and hospital births in western Australia: 2002-2013. J Paediatr Child Health. 2017;53(Suppl 2):3-17. doi: 10.1111/jpc.13494_72

7. Sanfelice CFO, Shimo AKK. Social representations on home birth. Esc Anna Nery. 2015; 19(4):606-13 doi: 10.5935/1414-8145.20150081

8. MacDorman MF, Declercq E. Trends and state variations in out-of-hospital births in the United States, 20042017. Birth. 2019;46(2):279-88. doi: 10.1111/birt.12411

9. Ministério da Saúde (BR). Rede Interagencial de Informações para a Saúde. DATASUS. Nascimento por residência mãe por ano do nascimento segundo região/domicílio (2010-2017) [Internet]. 2017 [acesso em 2019 out 29]. Disponível em: http://tabnet.datasus.gov.br/cgi/tabcgi.exe?sinasc/cnv/nvuf.def

10. Conselho Federal de Enfermagem (COFEN). Parecer Técnico CN SM/COFEN NN003/2019 dispõe sobre a Regulação e Prática da Enfermagem Obstétrica no espaço do parto domiciliar planejado [Internet]. Brasília (DF): COFEN; 2019 [acesso em 2019 out 29]. Disponível em: http://www.cofen.gov.br/parecer-tecnico-cnsmcofen-no-003-2019_74671.html

11. Moscovici S. Representações sociais: investigações em psicologia social. Petrópolis: Vozes; 2003.

12. Silva SED, Santos AL, Costa JL, Cunha NMF, Araújo JS, Moura AAA, et al. A teoria das representações sociais sob a ótica das pesquisas de enfermagem no Brasil. J Health Biol Sci. 2017;5(3):272-6 doi: 10.12662/23173076jhbs.v5i3.1319.p272-276.2017

13. Nascimento LCN, Souza TV, Oliveira ICS, Moraes JRMM, Aguiar RCB, Silva LF. Theoretical saturation in qualitative research: an experience report in interview with schoolchildren. Rev Bras Enferm. 2018;71(1):228-33. doi: 10.1590/0034-7167-2016-0616

14. Naderifar M, Goli H, Ghaljaie F. Snowball Sampling: a purposeful method of sampling in qualitative research. Strides Dev Med Educ. 2017;14(3):e67670. doi: 10.5812/sdme.67670

15. Bardin L. Análise de conteúdo. São Paulo: Edições 70; 2011.

16. Ministério da Saúde (BR), Secretaria de Ciência, Tecnologia e Insumos Estratégicos, Departamento de Gestão e Incorporação de Tecnologias em Saúde. Diretrizes nacionais de assistência ao parto normal: versão resumida. Brasília (DF): Ministério da Saúde; 2017. 
19 | Cunha IVA, Mata JAL, Fernandes LCR, Tanaka EZ, Sanfelice CFO.

17. Campbell K, Carson G, Azzam H, Hutton E. Statement of planned homebirth. J Obstet Gynaecol Can. 2019;41(2):223-7. doi: 10.1016/j.jogc.2018.08.008

18. The Royal Australian and New Zealand College of Obstetricians and Gynaecologists (RANZCOG). Home Births [Internet]. Melbourne: RANZCOG; 2017 [cited 2020 Oct 15]. Available from: https://ranzcog.edu.au/RANZCOG_SITE/media/RANZCOG-

MEDIA/Women\%27s\%20Health/Statement\%20and\%20guidelines/Clinical-Obstetrics/Home-Births-(C-Obs-2)Review-July-17.pdf?ext=.pdf

19. Santos EVM, Lima CB. Parto domiciliar assistido: abordando a atuação do enfermeiro obstetra. Temas Saúde [Internet]. 2018 [acesso em 2020 out 15];18(1):192-203. Disponível em: https://temasemsaude.com/wpcontent/uploads/2018/04/18110.pdf

20. Mata JALM. Enfermagem obstétrica no parto domiciliar planejado: responsabilidade legal e organização do serviço. In: Associação Brasileira de Enfermagem (ABEn), Associação Brasileira de Obstetrizes e Enfermeiros. Obstetras (Abenfo). PROENF: Programa de atualização em enfermagem: saúde materna e neonatal: ciclo 8. Porto Alegre: Artmed Panamericana; 2017. p. 75-125.

21. Conselho Regional de Enfermagem de Santa Catarina (COREN/SC). Parecer técnico COREN/SC nº 023/CT/2016. Parto Domiciliar Planejado. Florianópolis: COREN/SC; 2017 [acesso em 2020 out 15]. Disponível em: http://www.corensc.gov.br/wp-content/uploads/2017/01/PT-023-2016-Parto-Domiciliar-Planejado.pdf

22. Conselho Federal de Medicina (CFM). Recomendação 1/2012. Recomenda que a realização do parto ocorra em ambiente hospitalar de forma preferencial por ser mais segura. Brasília (DF): CFM; 2012. Disponível em: https://portal.cfm.org.br/images/Recomendacoes/1_2012.pdf

23. Pascoto GS, Tanaka EZ, Fernandes LCR, Shimo AKK, Sanfelice CFO. Dificuldades da assistência ao parto domiciliar na ótica de enfermeiras obstetras. Rev Baiana Enferm. 2020; 34:e36633 doi: 10.18471/rbe.v34.36633

24. Nygaard SS, Kesmodel US. Home births: here are we heading? Acta Obstet Gynecol Scand. 2018;97:1155-6 doi: https://doi.org/10.1111/aogs.13441

25. Reitsma A, Simioni J, Brunton G, Kaufman K, Hutton EK. Maternal outcomes and birth interventions among women who begin labour intending to give birth at home compared to women of low obstetrical risk who intend to give birth in hospital: a systematic review and meta-analyses. EClinicalMedicine. 2020;21:100319. doi: 10.1016/j.eclinm.2020.100319

26. Taheri M, Takian A, Taghizadeh Z, Jafari N, Sarafraz N. Creating a positive perception of childbirth experience: systematic review and meta-analysis of prenatal and intrapartum interventions Reprod Health. 2018;15:73. doi: 10.1186/s12978-018-0511-x

27. Leal MC, Bittencourt SA, Esteves-Pereira AP, Ayres BVS, Silva LBRAA, Thomaz EBAF, et al. Avanços na assistência ao parto no Brasil: resultados preliminares de dois estudos avaliativos. Cad Saúde Pública. 2019;35(7):e00223018. doi: 10.1590/0102-311x00223018

28. Rowe RE, Townend J, Brocklehurst P, Knight M, Macfarlane A, McCourt C, et al. Duration and urgency of transfer in births planned at home and in freestanding midwifery units in England: secondary analysis of the Birthplace national prospective cohort study. BMC Pregnancy Childbirth. 2013;13:224. doi: 10.1186/1471-2393$13-224$ 
Representações sociais de profissionais de saúde da área hospitalar sobre o parto... $\mid 20$

29. Cross-Sudworth F, Hindley J, Cheatham C, Clarke P, McAgree T. Creating a dedicated homebirth service: results of a 3-year pilot. Br J Midwifery. 2018;26:3. doi: 10.12968/bjom.2018.26.3.164

Editora Científica Chefe: Cristiane Cardoso de Paula

Editora associada: Graciela Dutra Sehnem

Fomento: Conselho Nacional de Desenvolvimento Científico e Tecnológico (CNPq)

\author{
Autor correspondente \\ Clara Fróes de Oliveira Sanfelice \\ E-mail: clarafos@unicamp.br \\ Endereço: Av. Constante Pavan, 2060 Residencial Porto Alegre, Casa 92, Betel, Paulínia-SP. CEP: 13148-198
}

\title{
Contribuições de Autoria
}

1 - Isabela Venturini Ayres Cunha

Concepção do estudo, análise/interpretação dos dados e revisão final com participação crítica e intelectual no manuscrito.

2 - Júnia Aparecida Laia da Mata

Análise/interpretação dos dados e revisão final com participação crítica e intelectual no manuscrito.

3 - Luciane Cristina Rodrigues Fernandes

Revisão final com participação crítica e intelectual no manuscrito.

4 - Erika Zambrano Tanaka

Revisão final com participação crítica e intelectual no manuscrito.

5 - Clara Fróes de Oliveira Sanfelice

Concepção do estudo, análise/interpretação dos dados e revisão final com participação crítica e intelectual no manuscrito.

\section{Como citar este artigo}

Cunha IVA, Mata JAL, Fernandes LCR, Tanaka EZ, Sanfelice CFO. Social representations of health professionals in the hospital area about planned home birth. Rev. Enferm. UFSM. 2021 [Acesso em: Anos Mês Dia]; vol.11 e66: 1-20. DOI: https://doi.org/10.5902/2179769263786 